\title{
The application of a combination of weighted least- squares and autoregressive methods in predictions of polar motion parameters
}

\author{
Fei Wu ${ }^{1} \cdot$ Kazhong Deng ${ }^{1} \cdot$ Guobin Chang ${ }^{1} \cdot$ Qianxin Wang ${ }^{1}$
}

Received: 7 May 2017/Accepted: 5 March 2018/Published online: 19 March 2018

(C) Akadémiai Kiadó 2018

\begin{abstract}
This study employs a combination of weighted least-squares extrapolation and an autoregressive model to produce medium-term predictions of polar motion (PM) parameters. The precisions of PM parameters extracted from earth orientation parameter (EOP) products are applied to determine the weight matrix. This study employs the EOP products released by the analysis center of the 'International Global Navigation Satellite System Service and International Earth Rotation and Reference Systems Service' needs to be modified to 'International Global Navigation Satellite System Service (IGS) and International Earth Rotation and Reference Systems Service (IERS)' as primary data. The polar motion parameters and their precisions are extracted from the EOP products to predict the changes in polar motion over spans of 1-360 days. Compared with the combination of least-squares and autoregressive model, this method shows considerable improvement in the prediction of PM parameters.
\end{abstract}

Keywords Earth orientation products - Weighted least-squares · Autoregressive model $\cdot$ Polar motion prediction

\section{Introduction}

Earth orientation parameters (EOPs) include the nutation and precession parameters, the polar motion parameters and the length of day (LOD). EOPs contain a wealth of geodynamics information and play a significant role in applications including the determination of high-precision satellite orbits, spacecraft tracking, laser measurements, and deep space exploration. These parameters are needed to achieve mutual conversion between celestial and terrestrial reference frames. Thanks to developments in modern measurement

Kazhong Deng

kzdeng@cumt.edu.cn

1 School of Environment Science and Spatial Informatics, China University of Mining and Technology, Xuzhou 221116, China 
techniques, such as very long baseline interferometry (VLBI), satellite laser ranging (SLR), global navigation satellite system (GNSS), and doppler orbitography by radio-positioning integrated on satellite (DORIS) (Dow et al. 2005), increasingly accurate observations of EOPs promote advances in celestial dynamics.

Due to the complexity of the data processing involved, EOP products cannot be calculated in real time using the data obtained by modern earth observation technologies. Thus, the parameters are usually provided after a delay of hours to days. However, for some practical applications, the EOPs must be acquired in advance. For example, in satellite navigation systems, only long-term predictions of EOPs can be used to achieve mutual conversion between the celestial and terrestrial reference frames when satellites enter the autonomous orbit mode. Therefore, a reliable and high precision prediction model is needed. However, EOPs are affected by many factors (Völgyesi 2006) that can be divided into two groups, specifically the effects of (1) different levels and periodic excitation sources and (2) high-frequency variations. These factors cause enormous challenges in the precise prediction of EOPs.

To check the accuracy of different methods of predicting EOPs, the Institute of Geodesy and Geophysics of the University of Vienna conducted two prediction competitions, the Earth Orientation Parameters Prediction Comparison Campaign (EOP PCC) in October 2005 (http://users.cbk.waw.pl/ kalma/EOP_PCC/) and the Earth Orientation Parameters Combination of Prediction Pilot Project (EOPCPPP) in October 2010 (http://eopcppp.cbk. waw.pl). The purposes of these activities were to encourage scholars worldwide to utilize different methods to predict EOPs and to assess the forecasting accuracy and applicability of various prediction methods. The prediction methods can be divided into two categories, specifically single models (including both linear and nonlinear models) and combinations of multi models (Freedman et al. 1994; Schuh et al. 2002; Kosek et al. 2004, 2008; Xu 2012; Guo et al. 2013; Xu and Zhou 2015). One major conclusion that was reached as a result of these competitions is that no single prediction technique is suitable for all EOPs over their entire ranges of variation. The following two conclusions were also reached. (1) Least-squares extrapolation of harmonic models and autoregressive prediction (LS + AR), spectral analysis and LS extrapolation, and neural networks display relatively good performance in predicting PM. (2) Kalman filters, wavelet decomposition and auto-covariance prediction, and adaptive transformation of the atmospheric angular momentum (AAM) yield relatively high-quality results in predicting UT1-UTC and LOD (Kalarus et al. 2007, 2010).

Many scholars have carried out studies on methods of predicting polar motion (Zhang 2012; Sun 2013; Lei 2016). Of these methods, LS + AR models represent a relatively stable type of model that is used to predict PM parameters; moreover, many scholars have made different improvements to this method (Sun and Xu 2012; Xu et al. 2012a, b; Yao et al. 2013). Note that the high calculation precision of the PM parameters is almost negligible compare with the prediction accuracy; thus, the effects of model error on the predictions should be emphasized. Therefore, how to address the residuals that result from fitting LS models to data has been examined by different scholars. Differential method processing LS + AR models are used mainly in short-term forecasts (Yan and Yao 2012), whereas WLS + AR models are employed in medium-and-long-term predictions of PM parameters. The main principle used by previous studies to construct the weight matrix is that relatively high weights are assigned when the predicted values lie close to the data; although this practice effectively improves the prediction accuracy, it depends considerably on the experience of the operator (Sun and $\mathrm{Xu}$ 2012). Therefore, in this paper, we propose employing the calculation precision of the PM parameters extracted from EOP 
products as weighting factors to determine the weight matrices of the vectors of observation used in the least-squares extrapolation model. In this study, the fitting parameters of the weighted least-squares model are first calculated; the AR model parameters are then determined from the residuals; and finally, the extrapolated values obtained from the WLS and AR models are combined to obtain the final predictions. Having extracted PM parameters and their precisions from the data products published by the analysis centres of the IGS and the IERS, which are taken to represent basic data, this study predicts mediumterm changes in PM and compares them with the results predicted by the LS + AR model to verify the feasibility of the method presented here.

\section{Methods}

\subsection{Weighted least-squares model}

The method is based on LS + AR. An introduction to the construction of and the calculations performed by the model is first presented. Existing studies have shown that the major trends of the PM contain a linear term and periodic terms, including the Chandler wobble and the annual and semi-annual terms (Sun 2013). The fitting equation of the LS model can be expressed as:

$$
\begin{aligned}
\mathrm{X}(t)= & a_{0}+a_{1} \cdot t+a_{2} \cdot \cos \left(\frac{2 \pi t}{T_{1}}\right)+a_{3} \cdot \sin \left(\frac{2 \pi t}{T_{1}}\right)+a_{4} \cdot \cos \left(\frac{2 \pi t}{T_{2}}\right)+a_{5} \cdot \sin \left(\frac{2 \pi t}{T_{2}}\right) \\
& +a_{6} \cdot \cos \left(\frac{2 \pi t}{T_{3}}\right)+a_{7} \cdot \sin \left(\frac{2 \pi t}{T_{3}}\right)
\end{aligned}
$$

where $\mathrm{X}(t)$ is the PM at time $t$; $t$ is the UTC time (in years); $a_{0}, a_{1}, a_{2}, a_{3}, a_{4}, a_{5}, a_{6}, a_{7}$ need to be estimated; $T_{1}, T_{2}, T_{3}$ are the periods of the semi-annual, annual and Chandler wobble terms, respectively. In this paper, $T_{1}=0.5 a, T_{2}=1 a$ and $T_{3}=1.183 a$. Estimates of the model parameters can be calculated using formula (2):

$$
\begin{aligned}
& \alpha=\left(A^{T} P A\right)^{-1} A^{T} P L \\
& \alpha=\left[\begin{array}{llllllll}
a_{0} & a_{1} & a_{2} & a_{3} & a_{4} & a_{5} & a_{6} & a_{7}
\end{array}\right]^{T} \\
& \mathrm{~L}=\left[\begin{array}{llll}
X\left(t_{1}\right) & X\left(t_{2}\right) & \cdots & X\left(t_{n}\right)
\end{array}\right]^{T}
\end{aligned}
$$

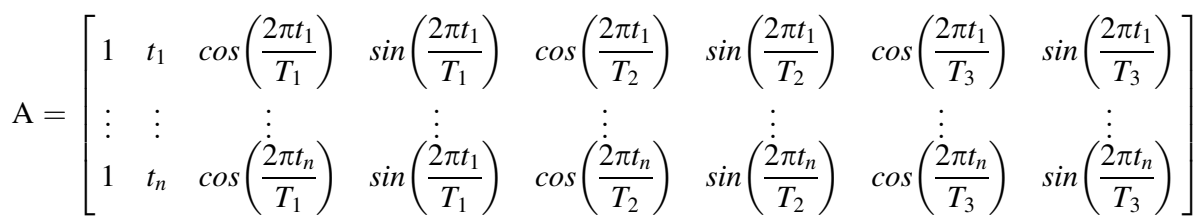

where $\alpha$ is the vector of estimated parameter; $A$ is a coefficient matrix; $L$ is a vector of observation; and $P$ is the weight matrix, which is a diagonal matrix. The residuals are then processed using an AR model. 


\subsection{Determination of the weight matrix}

The key feature of the WLS method is that it incorporates a weight matrix into the estimation of parameters based on LS models. An appropriate weighting method effectively improves the precision of the model parameters, thus resulting in a more stable base sequence for the AR model. In the actual process of surveying adjustment, using the calculated precision of the parameters as the posterior factor is a commonly weighting method. Therefore, this paper extracts the precision of PM parameters from the EOP products released by different analysis centers for use as a variance factor in building the weight matrix. The weight matrix is expressed as follows:

$$
\mathrm{P}_{w}=\left[\begin{array}{cccc}
\frac{\sigma_{0}^{2}}{\sigma_{1, w}^{2}} & 0 & 0 & 0 \\
0 & \frac{\sigma_{0}^{2}}{\sigma_{2, w}^{2}} & 0 & 0 \\
\vdots & \vdots & \ddots & \vdots \\
0 & 0 & 0 & \frac{\sigma_{0}^{2}}{\sigma_{n, w}^{2}}
\end{array}\right]
$$

where $w$ denotes the polar motion (PMX, PMY); $n$ is the length of the basic data; the unit weight variance $\sigma_{0}^{2}$ is assigned a value of 1 ; and $\sigma_{n, w}^{2}$ represents the variances of the calculated PM parameters on the $n$th day.

\subsection{AR model}

An AR model describes the relationship among the variables of a random series $z_{t}(t=1,2, \ldots N)$, which can be expressed as follows:

$$
z_{t}=\sum_{i=1}^{p} \varphi_{i} z_{t-i}+\varepsilon_{t}
$$

where $\varphi_{1}, \varphi_{2}, \ldots \varphi_{p}$ are autoregressive coefficients obtained using the least squares method; $p$ is the order of the model; and $\varepsilon$ represents zero-mean white noise. In this paper, the AR model is constructed by the fitting residuals of the WLS model.

A key step in applying AR model is determining the order $p$. Three main methods of estimating the orders of AR model exist. Specifically, these rules are the final prediction error (FPE) criterion, the akaike information criterion (AIC), and the criterion autoregressive transfer (CAT). In practice, these three methods are virtually equivalent to each other. The FPE criterion is adopted to determine the order of AR model.

$$
\operatorname{FPE}(p)=\frac{(N+p)}{(N-p)} P_{p}
$$

where

$$
P_{p}=\frac{1}{N-p} \sum_{t=p+1}^{N}\left(z_{t}-\sum_{j=1}^{p} \varphi_{j} z_{t-j}\right)^{2}
$$


Here $p=1,2, \ldots N$, and $P_{p}$ is the residual mean squared deviation of the model fitting sequence in $\mathrm{AR}(\mathrm{p})$. When FPE (p) reaches its minimum value, $\mathrm{p}$ is taken to represent the order of the best model.

\subsection{Prediction error estimates}

The mean absolute error (MAE) is an indicator of prediction accuracy. It is calculated as:

$$
\operatorname{MAE}_{i}=\frac{1}{n} \sum_{j=1}^{n}\left|S_{j}^{i}-O_{j}^{i}\right|
$$

where $i$ is the prediction interval; $S_{j}^{i}, O_{j}^{i}$ are the predicted and released values, respectively, on day $\mathrm{j}$; and $n$ is the number of predictions used to calculate the statistics.

\section{Examples and analysis}

The products released by IGS and IERS contain the values and precision of PM parameters, and the time interval used in the products is 1 day. However, the EOP products released by the IGS Analysis Centre are determined using GNSS technology, whereas those of the IERS Analysis Centre are calculated using multiple technologies (such as GNSS, VLBI, SLR, and DORIS); the precisions of the PM parameters are not the same. In this study, the EOP products released by IGS and IERS are used as basic experimental data to predict the medium-term PM and to verify the effectiveness of the method proposed. As recent studies have shown (Sun et al. 2015), the highest accuracies are obtained in predictions using of the LS + AR models when an input sequence of 10 years is used. Therefore, this thesis takes 10 years as an elementary sequence to predict PM using an interval of 1 day and a span of 1-360 days.

\subsection{Experiments based on products released by IGS}

The values and the precisions derived from the PM parameters products issued by IGS (ftp://cddis.gsfc.nasa.gov/pub/gps/products/), which extend from January 1, 2002 to December 31, 2011, are employed to construct the prediction model and build the weight matrix. For each prediction (1-360 days), 600 and 1000 experiments are performed using the LS + AR and WLS + AR methods. The predictions extend from January 1, 2012 to August 18, 2014 and January 1, 2012 to September 22, 2015, and the sampling interval is 1 day. Tables 1 and 2 list the MAE values for the different prediction intervals, and these values are shown graphically in Figs. 1 and 2. 
Table 1 Statistical accuracy results in 600 IGS forecasts

\begin{tabular}{|c|c|c|c|c|c|c|}
\hline \multirow{2}{*}{$\begin{array}{l}\text { MAE } \\
\text { Prediction } \\
\text { day }\end{array}$} & \multicolumn{3}{|c|}{ PMX (mas) } & \multicolumn{3}{|c|}{ PMY (mas) } \\
\hline & $\mathrm{LS}+\mathrm{AR}$ & $\mathrm{WLS}+\mathrm{AR}$ & $\begin{array}{l}\text { Accuracy } \\
\text { improvement (\%) }\end{array}$ & $\mathrm{LS}+\mathrm{AR}$ & $\mathrm{WLS}+\mathrm{AR}$ & $\begin{array}{l}\text { Accuracy } \\
\text { improvement (\%) }\end{array}$ \\
\hline 1 & 0.216 & 0.215 & 0.074 & 0.106 & 0.106 & 0 \\
\hline 5 & 1.484 & 1.480 & 0.23 & 0.665 & 0.660 & 0.739 \\
\hline 10 & 2.831 & 2.793 & 1.353 & 1.244 & 1.226 & 1.51 \\
\hline 30 & 8.210 & 8.024 & 2.26 & 3.875 & 3.613 & 6.765 \\
\hline 60 & 15.886 & 15.106 & 4.907 & 8.114 & 7.022 & 13.467 \\
\hline 100 & 23.285 & 20.704 & 11.083 & 14.159 & 10.262 & 27.525 \\
\hline 120 & 27.145 & 22.273 & 17.95 & 16.218 & 11.193 & 30.986 \\
\hline 150 & 31.700 & 24.281 & 23.405 & 17.826 & 12.486 & 29.957 \\
\hline 200 & 33.499 & 25.094 & 25.092 & 15.553 & 11.851 & 23.803 \\
\hline 250 & 30.509 & 23.914 & 21.616 & 13.475 & 9.726 & 27.819 \\
\hline 300 & 30.179 & 25.690 & 14.874 & 15.579 & 10.894 & 30.069 \\
\hline 360 & 36.631 & 25.753 & 29.697 & 18.685 & 13.376 & 28.413 \\
\hline
\end{tabular}

Table 2 Statistical of accuracy results in 1000 IGS forecasts

\begin{tabular}{|c|c|c|c|c|c|c|}
\hline \multirow{2}{*}{$\begin{array}{l}\text { MAE } \\
\text { Prediction } \\
\text { day }\end{array}$} & \multicolumn{3}{|c|}{ PMX (mas) } & \multicolumn{3}{|c|}{ PMY (mas) } \\
\hline & $\mathrm{LS}+\mathrm{AR}$ & $\mathrm{WLS}+\mathrm{AR}$ & $\begin{array}{l}\text { Accuracy } \\
\text { improvement (\%) }\end{array}$ & $\mathrm{LS}+\mathrm{AR}$ & $\mathrm{WLS}+\mathrm{AR}$ & $\begin{array}{l}\text { Accuracy } \\
\text { improvement (\%) }\end{array}$ \\
\hline 1 & 0.212 & 0.211 & 0.308 & 0.175 & 0.174 & 0.06 \\
\hline 5 & 1.482 & 1.457 & 1.695 & 1.092 & 1.081 & 0.978 \\
\hline 10 & 2.831 & 2.720 & 3.913 & 2.056 & 2.024 & 1.567 \\
\hline 30 & 8.380 & 7.818 & 6.709 & 6.255 & 5.875 & 6.072 \\
\hline 60 & 16.666 & 14.619 & 12.279 & 13.667 & 11.713 & 14.295 \\
\hline 100 & 25.904 & 20.023 & 22.702 & 24.593 & 18.373 & 25.289 \\
\hline 120 & 29.763 & 21.616 & 27.372 & 28.483 & 20.576 & 27.758 \\
\hline 150 & 33.988 & 23.419 & 31.097 & 32.161 & 22.873 & 28.88 \\
\hline 200 & 35.434 & 23.472 & 33.759 & 30.309 & 21.760 & 28.208 \\
\hline 250 & 34.594 & 23.404 & 32.347 & 28.380 & 20.871 & 26.459 \\
\hline 300 & 35.652 & 27.121 & 23.928 & 33.023 & 24.328 & 26.33 \\
\hline 360 & 41.881 & 29.845 & 28.739 & 39.246 & 28.954 & 26.224 \\
\hline
\end{tabular}




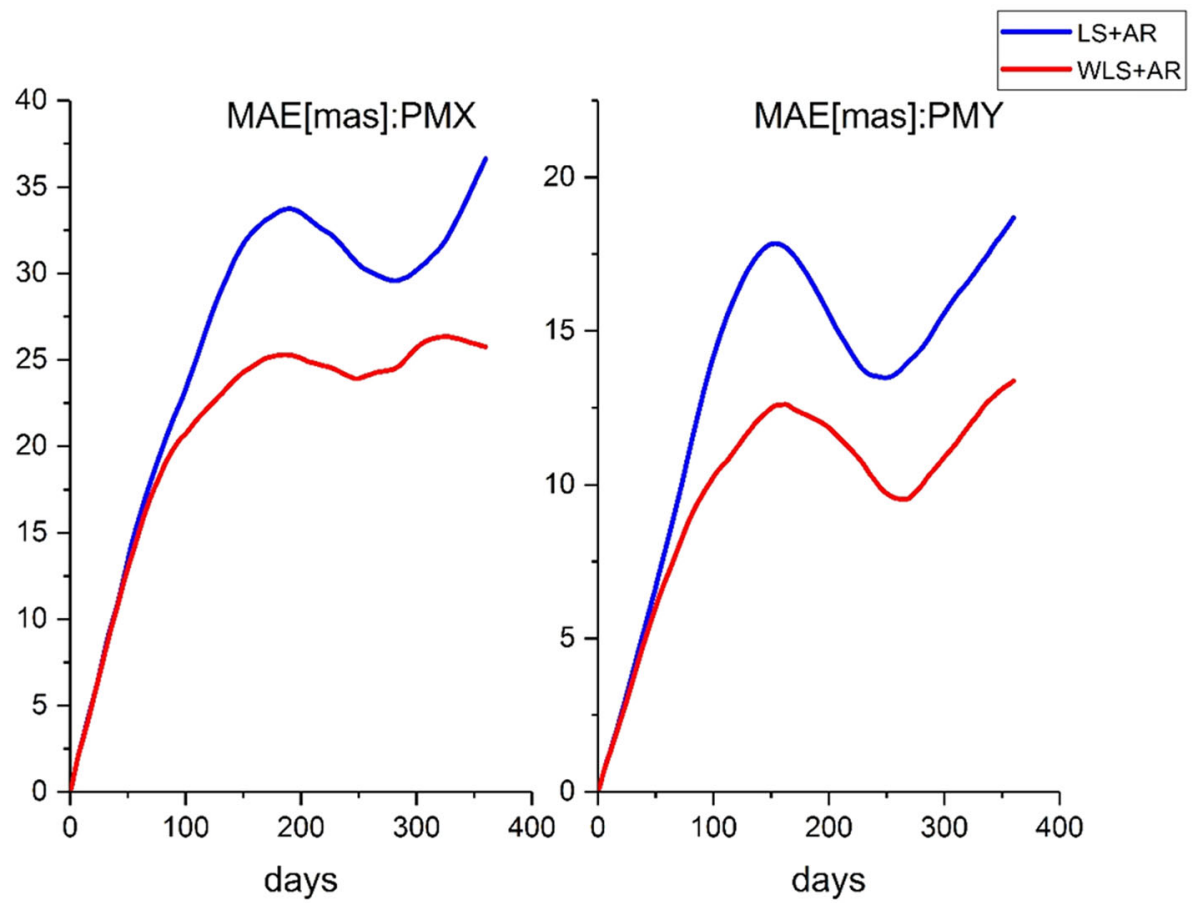

Fig. 1 MAE values calculated for 600 IGS forecasts over different intervals

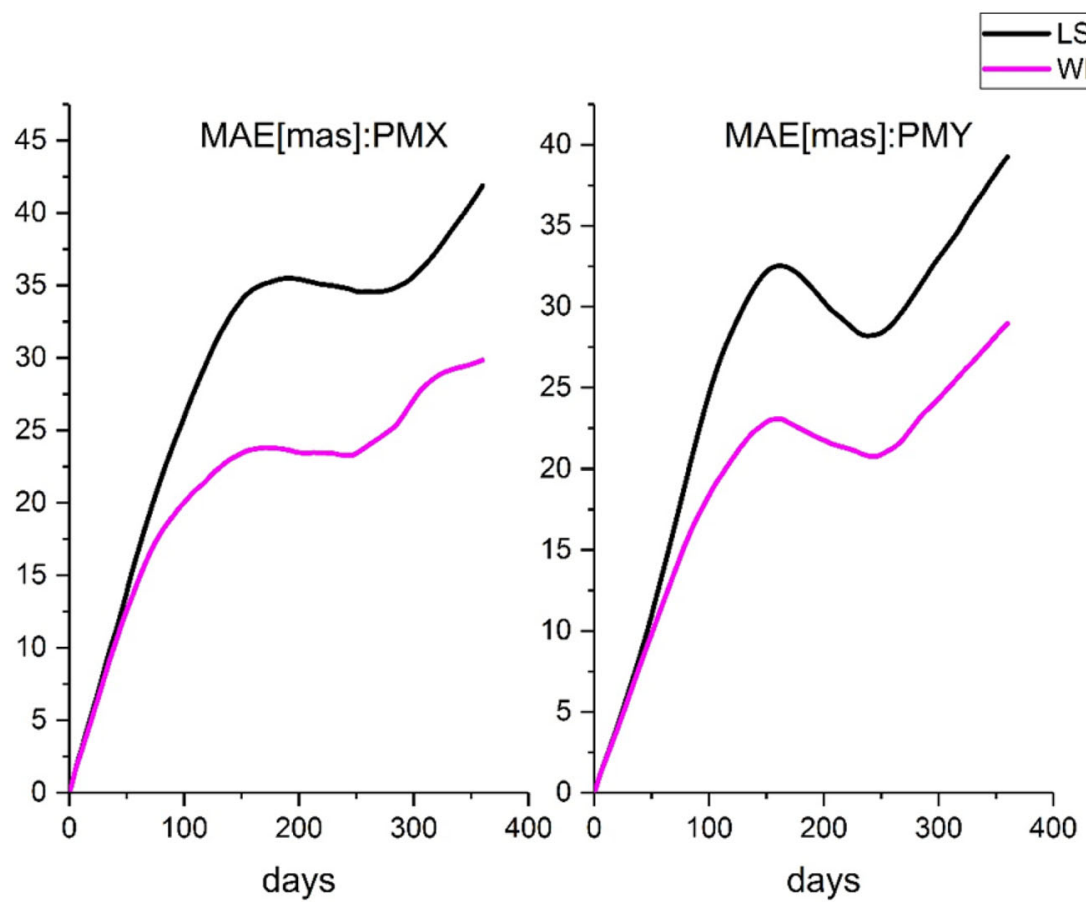

Fig. 2 MAE values calculated for 1000 IGS forecasts over different intervals 


\subsection{Experiments based on products released by IERS}

As another example, this study chooses the values and the precision of PM parameters issued by IERS 08 C04 EOP product (ftp://ftp.iers.org/products/eop/long-term/c04_08/ iau2000/), which extends from January 1, 2002 to December 31, 2011. These data are used to construct the prediction model and build the weight matrix. 600 and 1000 experiments are performed using the LS + AR and WLS + AR methods for each prediction (1-360 days), and the sampling interval is uniformly 1 day. The prediction dates are the same as the previous two experiments. The results are presented in Tables 3, 4 and Figs. 3, 4.

Table 3 Statistical of accuracy results in 600 IERS forecasts

\begin{tabular}{|c|c|c|c|c|c|c|}
\hline \multirow{2}{*}{$\begin{array}{l}\text { MAE } \\
\text { Prediction } \\
\text { day }\end{array}$} & \multicolumn{3}{|c|}{ PMX (mas) } & \multicolumn{3}{|c|}{ PMY (mas) } \\
\hline & $\mathrm{LS}+\mathrm{AR}$ & $\mathrm{WLS}+\mathrm{AR}$ & $\begin{array}{l}\text { Accuracy } \\
\text { improvement }(\%)\end{array}$ & $\mathrm{LS}+\mathrm{AR}$ & $\mathrm{WLS}+\mathrm{AR}$ & $\begin{array}{l}\text { Accuracy } \\
\text { improvement }(\%)\end{array}$ \\
\hline 1 & 0.224 & 0.223 & 0.23 & 0.108 & 0.108 & 0 \\
\hline 5 & 1.492 & 1.479 & 0.88 & 0.662 & 0.654 & 1.182 \\
\hline 10 & 2.895 & 2.836 & 2.018 & 1.237 & 1.213 & 1.974 \\
\hline 30 & 8.785 & 8.190 & 6.77 & 3.873 & 3.540 & 8.587 \\
\hline 60 & 17.603 & 15.323 & 12.95 & 8.143 & 6.996 & 14.091 \\
\hline 100 & 25.168 & 20.725 & 17.653 & 14.207 & 11.114 & 21.774 \\
\hline 120 & 28.619 & 21.680 & 24.246 & 16.265 & 12.706 & 21.879 \\
\hline 150 & 33.230 & 24.343 & 26.744 & 17.840 & 13.974 & 21.673 \\
\hline 200 & 36.156 & 24.995 & 30.869 & 15.459 & 12.271 & 20.623 \\
\hline 250 & 33.790 & 24.508 & 27.468 & 13.287 & 9.564 & 28.018 \\
\hline 300 & 32.471 & 25.898 & 20.242 & 15.427 & 11.918 & 22.747 \\
\hline 360 & 37.064 & 26.997 & 27.161 & 18.691 & 15.557 & 16.765 \\
\hline
\end{tabular}

Table 4 Statistical of accuracy results for 1000 IERS forecasts

\begin{tabular}{|c|c|c|c|c|c|c|}
\hline \multirow{2}{*}{$\begin{array}{l}\text { MAE } \\
\text { Prediction } \\
\text { day }\end{array}$} & \multicolumn{3}{|c|}{ PMX (mas) } & \multicolumn{3}{|c|}{ PMY (mas) } \\
\hline & $\mathrm{LS}+\mathrm{AR}$ & $\mathrm{WLS}+\mathrm{AR}$ & $\begin{array}{l}\text { Accuracy } \\
\text { improvement }(\%)\end{array}$ & $\mathrm{LS}+\mathrm{AR}$ & $\mathrm{WLS}+\mathrm{AR}$ & $\begin{array}{l}\text { Accuracy } \\
\text { improvement }(\%)\end{array}$ \\
\hline 1 & 0.224 & 0.223 & 0.526 & 0.183 & 0.183 & 0.024 \\
\hline 5 & 1.484 & 1.459 & 1.712 & 1.100 & 1.089 & 1.001 \\
\hline 10 & 2.842 & 2.738 & 3.659 & 2.054 & 2.027 & 1.299 \\
\hline 30 & 8.716 & 8.032 & 7.851 & 6.254 & 5.955 & 4.782 \\
\hline 60 & 17.459 & 15.250 & 12.652 & 13.656 & 12.518 & 8.329 \\
\hline 100 & 26.305 & 20.661 & 21.455 & 24.278 & 21.065 & 13.237 \\
\hline 120 & 29.746 & 22.268 & 25.141 & 28.008 & 24.067 & 14.068 \\
\hline 150 & 33.524 & 25.122 & 25.062 & 31.456 & 26.488 & 15.793 \\
\hline 200 & 34.771 & 25.490 & 26.691 & 30.076 & 25.681 & 14.611 \\
\hline 250 & 33.630 & 25.745 & 23.446 & 27.763 & 24.092 & 13.22 \\
\hline 300 & 36.098 & 30.768 & 14.765 & 31.855 & 28.775 & 9.67 \\
\hline 360 & 41.769 & 33.951 & 18.717 & 39.216 & 35.485 & 9.514 \\
\hline
\end{tabular}




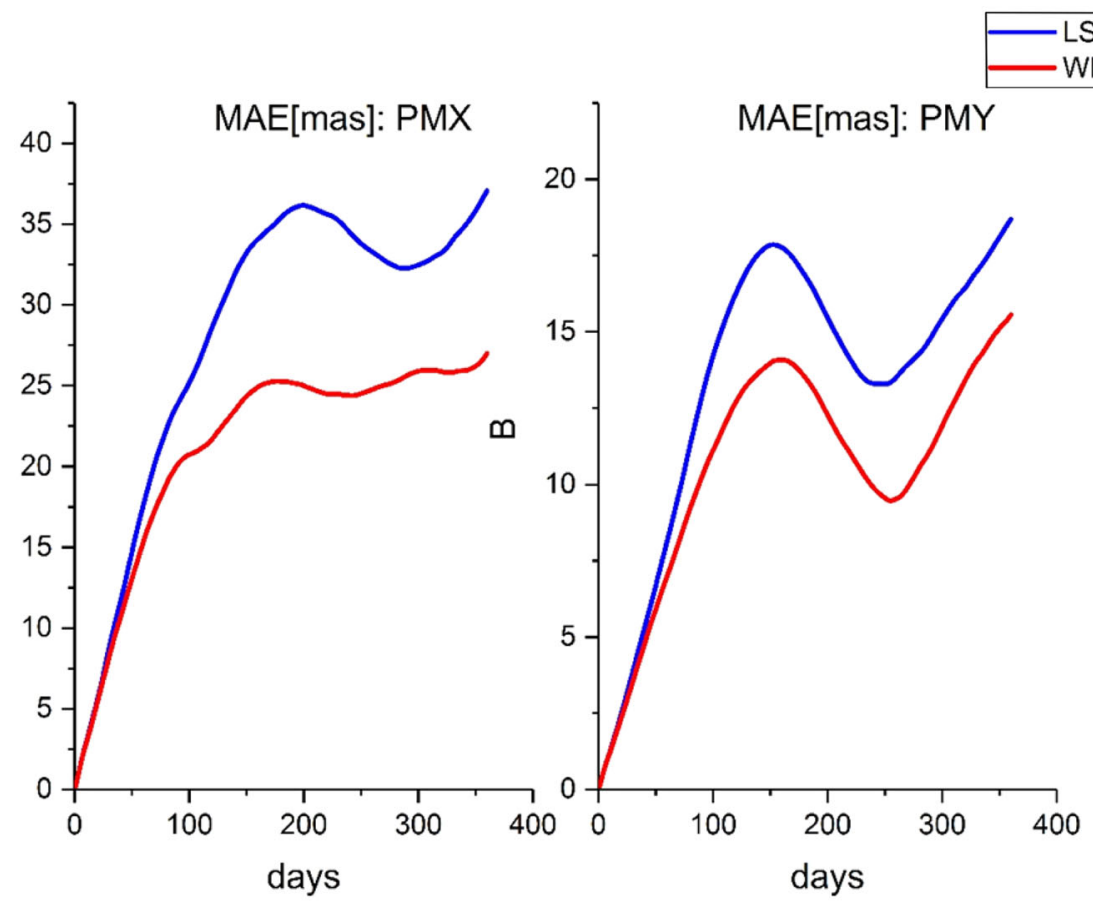

Fig. 3 MAE values calculated in 600 IERS forecasts

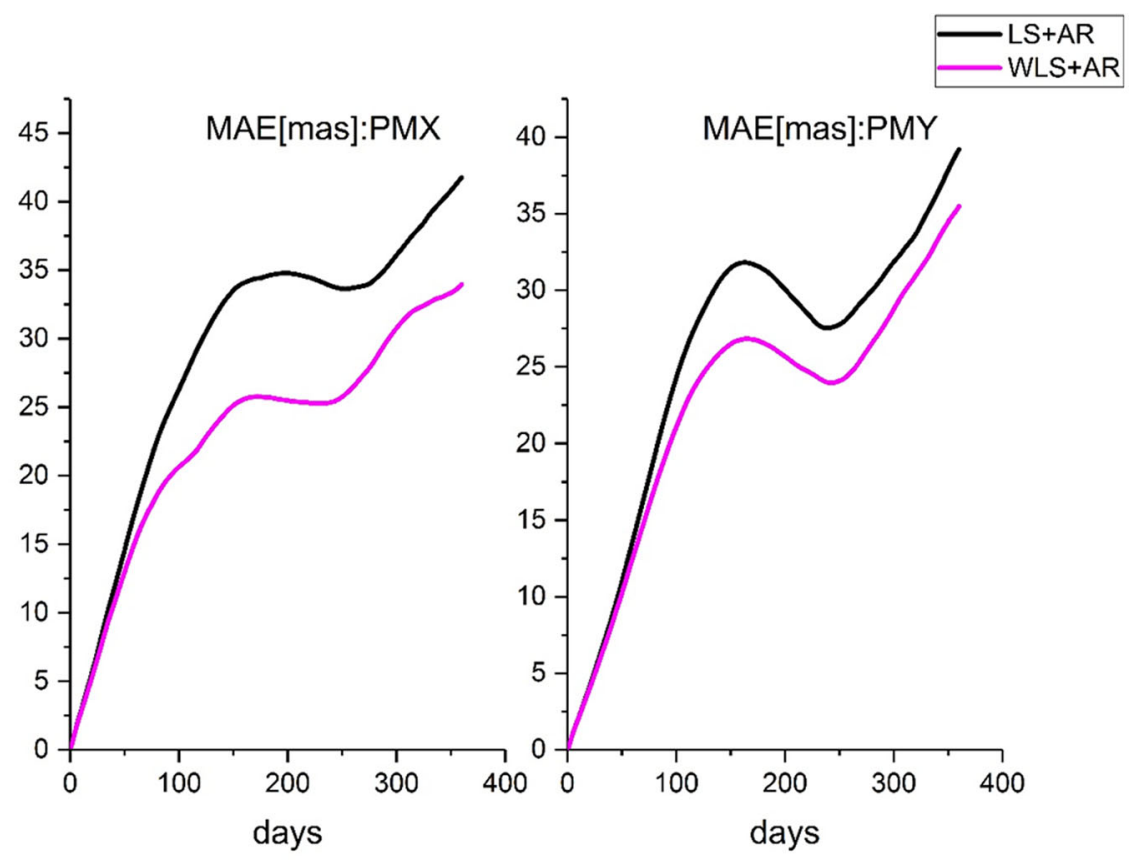

Fig. 4 MAE values calculated for 1000 IERS forecasts 
The above results indicate that the prediction accuracy of the WLS + AR model shows improvement at different levels in both the PMX and PMY directions. The improvements in the forecasting accuracy for short-term predictions (1-30 days in the future) are not obvious; the maximum improvement in the polar motion parameters in the PMX direction is less than $8.19 \%$, whereas that in the PMY direction is within $8.587 \%$. However, when the prediction span exceeds 30 days, the accuracy of the prediction displays clear improvements. For the group of 600 experiments, the prediction accuracy in the PMX direction calculated using the IGS and IERS data increases by an average of 16.09 and $21.43 \%$, whereas that in the PMY direction using the IGS and IERS data increases by 23.64 and $19.19 \%$, respectively. In the group of 1000 experiments, the prediction accuracy in the PMX direction calculated using the IGS and IERS data increases by an average of 24.14 and $19.04 \%$, whereas that in the PMY direction using the IGS and IERS data increases by 22.94 and $11.07 \%$, respectively. Compared with the LS + AR model, the WLS + AR model generally better in predicting polar motion parameters and yields more accurate medium-term forecasts.

\section{Conclusion}

This paper proposes a method of producing medium-term predictions of polar motion parameters using a WLS + AR model. In this method, the calculation precision of the PM parameters is employed to produce weighting factors to build the weight matrix of the vector of observations in the least-squares extrapolation model. More accurate parameters and extrapolated values of the LS model while and more stable residuals to form and calculate the AR model can be obtained using this method. The EOP products released by the analysis center of IGS and IERS are used as the basic data to predict the polar motion parameters in groups of 600 and 1000 experiments with a 1-day prediction interval and a span of 360 days. The results of the experiments show that, compared with the LS + AR model, the WLS + AR model proposed in this paper effectively improves the accuracy with which polar motion parameters can be predicted.

Acknowledgements The work is supported by the Ordinary University Graduate Student Research Innovation Project of Jiangsu Province (KYLX16_0541), National Natural Science Foundation of China (41774005), National Natural Science Foundation of China (41404033), Open research fund projects of the state key laboratory (SKLGIE2014-Z-1-1) and Project on Basic Research of The National Department of Science and Technology (2015FY310200). We thank the IERS and IIGS for providing the EOPs products.

\section{References}

Dow JM, Gurtner W, Schlüter W (2005) The IGGOS viewed from the space geodetic services. J Geodyn 40(4):375-386

Freedman AP, Steppe JA, Dickey JO, Eubanks TM, Sung LY (1994) The short-term prediction of universal time and length of day using atmospheric angular momentum. J Geophys Res Solid Earth 99(B4):6981-6996

Guo JY, Li YB, Dai CL, Shum CK (2013) A technique to improve the accuracy of earth orientation prediction algorithms based on least squares extrapolation. J Geodyn 70(10):36-48

Kalarus M, Kosek W, Schuh H (2007) Current results of the earth orientation parameters prediction comparison campaign. Proc Journées 84(10):587-596

Kalarus M, Schuh H, Kosek W, Akyilmaz O, Bizouard C, Gambis D et al (2010) Achievements of the earth orientation parameters prediction comparison campaign. J Geodesy 84(10):587-596 
Kosek W, McCarthy DD, Johnson TJ, Kalarus M (2004) Comparison of polar motion prediction results supplied by the IERS Sub-bureau for Rapid Service and Predictions and results of other prediction methods. In: Finkelstein A, Capitaine N (eds) Proc. Journées 2003 Systèmes de Référence SpatioTemporels, pp 164-169

Kosek W, Kalarus M, Niedzielski T (2008) Forecasting of the earth orientation parameters comparison of different algorithms. Nagoya J Med Sci 69(3-4):133-137

Lei Y (2016) Study on method for high accuracy prediction of earth rotation parameters. Chinese Academy of Sciences, Beijing

Schuh H, Ulrich M, Egger D, Müller J, Schwegmann W (2002a) Prediction of earth orientation parameters by artificial neural networks. J Geodesy 76(5):247-258

Sun Z (2013) Research on the theories and algorithms of earth rotation parameters prediction. Chang'an University, Xi'an

Sun Z, Xu T (2012) Prediction of earth rotation parameters based on improved weighted least squares and autoregressive model. Geodesy Geodyn 3(3):57-64

Sun Z, Xu T, He B, Ren G (2015) Prediction and analysis of Chinese earth rotation parameters based on robust least-squares and autoregressive model. In: China satellite navigation conference (CSNC) 2015 proceedings: vol II. Springer, Berlin, Heidelberg, pp 477-486

Völgyesi L (2006) Physical backgrounds of Earth's rotation, revision of the terminology. Acta Geod Geophys Hung 41(1):31-44

Xu XQ (2012) Researches on high accuracy prediction methods of earth orientation parameters. University of Chinese Academy of Sciences, Beijing

Xu XQ, Zhou YH (2015) EOP prediction using least square fitting and autoregressive filter over optimized data intervals. Adv Space Res 56(10):2248-2253

Xu XQ, Zotov L, Zhou YH (2012a) Combined prediction of earth orientation parameters. In: China satellite navigation conference (CSNC) 2012 proceedings lecture notes in electrical engineering, 2012, vol 160, Part 2, pp 361-369. https://doi.org/10.1007/978-3-642-29175-3_32

Xu XQ, Zhou YH, Liao XH (2012b) Short-term earth orientation parameters predictions by combination of the least-squares, ar model and Kalman filter. J Geodyn 62(1016):83-86

Yan F, Yao Y (2012) Short-term prediction methods and realization of earth rotation parameters. J Geod Geodyn 32(4):71-75

Yao YB, Yue SQ, Chen P (2013) A new LS + AR model with additional error correction for polar motion forecast. Sci China Earth Sci 56(5):818-828

Zhang H (2012) Researches on the prediction theories and algorithms of polar motion of earth orientation parameters. Central South University, Changsha 